\title{
HOMEOMORPHISMS OF A CERTAIN CUBE WITH HOLES $\left({ }^{1}\right)$
}

\author{
BY
}

\section{DONALD MYERS}

\begin{abstract}
For some manifolds the group of isotopy classes of self-homeomorphisms is known. In this paper this group is computed for a well-known cube with two holes. Two related manifolds are defined and the groups of isotopy classes on these manifolds are given without proof. One of these cubes with holes is such that every homeomorphism is isotopic to the identity.
\end{abstract}

1. Introduction. The group of homeomorphisms (modulo those isotopic to the identity) of a manifold onto itself is known for such manifolds as the $n$-cells and the solid torus. For closed surfaces, the generators are known [2]. We compute the group for the manifold $K$ and demonstrate ways to alter $K$ to obtain other manifolds with simpler homeomorphism groups.

Definition. $K$ is the closure of the complement in $S^{3}$ of a regular neighborhood of the 1-complex of Figure 2.

Theorem. The group of orientation-preserving bomeomorphisms of $K$ onto itself modulo the subgroup of bomeomorphisms isotopic to the identity bas the presentation $\left(f, g, r ; f g=g f, r^{2}, r f=g r\right)$.

Everything is assumed to be polyhedral. All homology groups have integral coefficients. The letters "scc" stand for "simple closed curve." Notation-wise, we usually do not distinquish between isotopic homeomorphisms. The term " $C$ homeomorphism" is as in [2a]. See [1] for much of the notation.

2. Homeomorphisms on $K$. Figures 1 and 2 define certain subsets of $K$ and elements of $\pi_{1}(K)$ respectively. Using the usual techniques for knots one easily shows that $\pi_{1}(K)$ has the presentation: $\left(a, d, g ; a^{-1} g^{-1}\right.$ agdad $\left.{ }^{-1}\right)$ and $H_{1}(K)=$ $(g, d ; g d=d g)$.

Lemma 1. $K$ is boundary incompressible (that is, any scc in $\partial K$ that bounds a disk in $K$ also bounds a disk in $\partial K$ ) and consequently every properly embedded disk in $K$ separates a 3-cell from $K$.

Received by the editors September 6, 1973.

AMS (MOS) subject classifications (1970). Primary 57A10; Secondary 55A25.

Key words and phrases. 3-manifolds, homeomorphisms, cubes with holes, $C$-homeomorphisms.

(1) This paper contains parts of a dissertation written at the University of lowa under Tom Price. 


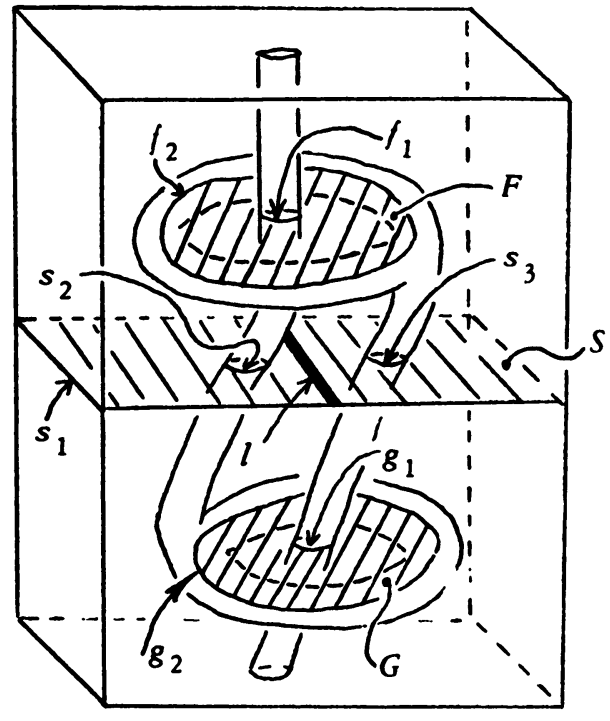

Figure 1

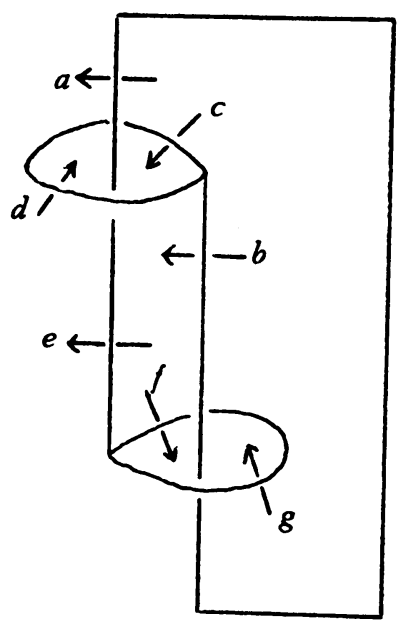

Figure 2

Proof. The latter statement follows from the first since $\partial K$ is connected and $K$ is embedded in $S^{3}$ which is separated by 2-spheres into two 3-cells. Suppose $K$ is not boundary incompressible. Then we can take a disk $D$ in general position with respect to $F$ and $G$ which contradicts the lemma and hits $F \cup G$ in as few components as possible. If a component of $(F \cup G) \cap D$ bounds a disk in $F \cup G$, then pick an interior such disk $E$. Cut $D$ at $\partial E$ and sew $E$ onto it and push off $F \cup G$, reducing the number of components of $(F \cup G) \cap D$. Thus any scc in $(F \cup G) \cap D$ is parallel in $F \cup G$ to a boundary component of $F \cup G$. If $u$ is such a component, say in $F$, which is parallel via the annulus $A$ to $f_{1}$ in $F$, then take the disk $E^{\prime}$ bounded by $u$ in $D$. Thus $A \cup E^{\prime}$ shows that $f_{1}$ is null homotopic in $K$ which implies $a=1$ in $\pi_{1}(K)$ which contradicts the Freiheitsatz [3, p. 252].

If $(F \cup G) \cap D$ contains an arc then choose one, say $u$, so that if $E$ is one of the disks cut off $D$ by $u$ then $E-u$ misses $F \cup G$. Say $u \subseteq F$. Now $u$ cuts a disk off $F$ since otherwise it has one endipoint in $f_{1}$ and the other in $f_{2}$; that is, $\operatorname{cl}(\partial E-u)$ is an arc on $\partial K$ with one endpoint in $f_{1}$ and the other in $f_{2}$ which misses $G$, a contradiction since $g_{1}$ separates $\partial F$ in $\partial K$. Thus we can pick a disk $E^{\prime}$ which is cut off $F$ by $D$ whose interior misses $D$. Then $v=E^{\prime} \cap D$ cuts $D$ into two disks. Add $E^{\prime}$ to each of these disks to get two properly embedded disks. Since $D$ contradicts the lemma, at least one of these disks $D^{\prime}$ must also, and after pushing it off $F$ at $E^{\prime}$ we have that $(F \cup G) \cap D^{\prime}$ has fewer components than does $(F \cup G) \cap D$, a contradiction to the choice of $D$. Thus 
we conclude that $D$ misses $F$ and $G$, that is, $\partial D$ lies in $\partial K$ missing the $f_{i}$ and $g_{i}$ which cut $\partial K$ into disks with either one or two holes. The Schoenflies theorem then gives that $\partial D$ is parallel to one of the $f_{i}$ or $g_{i}$ and again $a=1$ in $\pi_{1}(K)$, a contradiction which completes the proof.

Lemma 2. (a) Let $A$ be an annulus in $K$ whose interior lies in int $K$, whose boundary components lie in $F$ and are parallel in $F$ to the components of $\partial F$, and whose interior misses $F$ and $G$. Then $A$ is parallel in $K$ to an annulus in F. (b) There does not exist an annulus $A$ with all the above properties except that the last property is amended to the extent that $A \cap G$ is a single scc parallel in $G$ to the components of $\partial G$ and in $A$ to the components of $\partial A$.

Proof. Take a regular neighborhood of $F$ in $K$ meeting $\partial K$ regularly. Let $N_{1}$ and $N_{2}$ be the closed complementary domains of $F$ in this neighborhood whose interiors hit $D_{1}$ and $D_{2}$ of Figure 3 respectively.

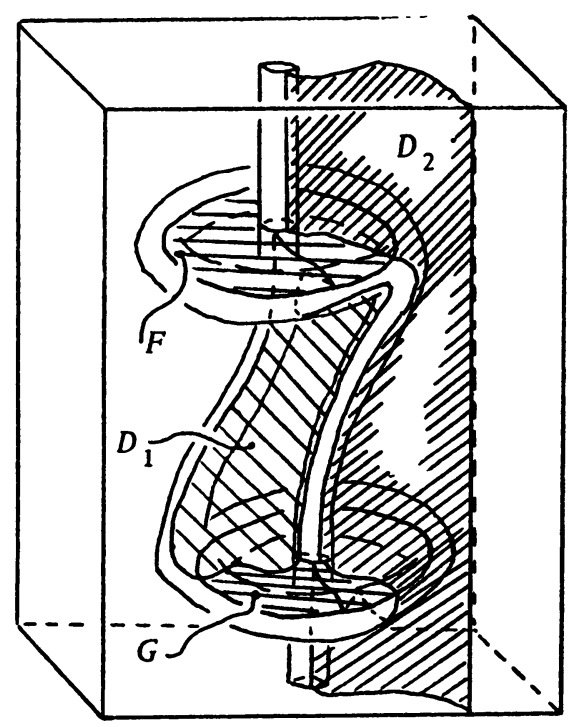

Figure 3

We have that the only components of $A \cap D_{i}$ are arcs with endpoints in $A \cap$ $(F \cup G)$ or scc's (or points in $F \cap \partial A$ ) since we may assume general positioning. Since the components of $\partial A$ are parallel in $F$ to $f_{1}$ we may assume that each component of $\partial A$ hits $D_{1} \cap F$ exactly once. In (b) we may similarly assume $A$ $\cap G$ hits $D_{1} \cap G$ exactly once and each of the two components of $D_{2} \cap G$ exactly once each. In (b) we conclude that the arc in $D_{1} \cap G$ with one endpoint in $G$ has the other in $F$. Since $D_{1} \cap A$ has only two components in $F$ and since $F \cap A=$ $\partial A$ the other component of $D_{1} \cap A$ is a point which is the endpoint of an arc in 
$D_{2} \cap A$. This is the only arc in $D_{2} \cap A$ with an endpoint in $F$. This arc has its other endpoint in $G$. But each of the components of $G \cap D_{2}$ has exactly one point in $A$. Thus $\partial D_{2}$ contains exactly three points which are endpoints of arcs in $D_{2}$. This is a contradiction and so (b) is established. For (a) we have that either $D_{1}$ $\cap A$ or $D_{2} \cap A$ consists of no arcs, say $D_{i}$. Any scc in $D_{i} \cap A$ bounds a disk in $D_{i}$ and since the components of $\partial A$ are parallel in $F$ to $f_{1}$ the scc must also bound a disk in $A$ since otherwise we would have $a=1$ in $\pi_{1}(K)$ as in Lemma 1. Thus we can push $A$ off $D_{i}$ at these scc's in the usual fashion. Then construct an isotopy which is the identity on $D_{i} \cup G \cup F \cup \partial K$ to push $A$ into $N_{j}(j \neq 1)$. Then we apply $[5$, p. 65] and reverse all isotopies.

Lemma 3. If $b$ is a homeomorphism of $K$ onto itself, then an isotopy allows the assumption that $b\left(s_{1}\right)=s_{1}$.

Proof. Since $\partial F \cup \partial G$ cuts $\partial K$ into disks with at most two holes it will suffice to show that $b\left(s_{i}\right)$ misses $\partial F \cup \partial G$ for some $i$. For in this case it is parallel in $\partial K$ to one of the components of $\partial F \cup \partial G$. Since $b\left(s_{i}\right)$ separates $\partial K$ it must be parallel to $f_{1}$ and $g_{1}$ and hence to $s_{1}$. 'Thus $b\left(s_{1}\right)$ is parallel to $s_{1}$ since both are parallel to $b\left(s_{i}\right)$. An isotopy now brings $b\left(s_{1}\right)$ to $s_{1}$. Supposing this is not the case, we let $R=b S \cap(F \cup G)$ be such that $b S$ is in general position with respect to $F \cup G$ and contains as few components as possible (apply the appropriate isotopies). The proof is in several steps.

Step 1. No scc in $R$ bounds a disk in $b S, F$, or $G$. (Such a scc in, say, $b S$ must necessarily bound a disk in $F$ or $G$ also since $a \neq 1$ in $\pi_{1}(K)$, as in the proof of Lemma 1. Push this scc off in the usual fashion.)

Step 2. No component of $R$ along with an arc in $\partial b S$ bounds a disk in $b S$. (If this is not the case then take a component $u$ which is innermost in $b S$ in the sense that if $D$ is the disk cut off $b S$ by $u$ in $R$ then $\partial D-u$ misses $F$ and $G$. By Step 1 int $D$ also misses $F$ and $G$. Since $c l(\partial D-u)$ lies in $\partial K$ and $f_{1}$ separates $\partial G$ in $\partial K$ and $g_{1}$ separates $\partial F$ in $\partial K$, both endpoints of $u$ lie in the same component of $\partial F \cup \partial G$, say in a component of $\partial F$. Then $u$ cuts a disk $D^{\prime}$ off $F$. Then $D \cup D^{\prime}$ is a properly embedded disk in $K$ and by Lemma 1 it separates a 3-cell off $K$. Use this 3-cell to construct an isotopy taking $D$ onto $D^{\prime}$ and then slightly to the other side. This reduces the number of components of $R$, a contradiction.)

Step 3. There are no scc's in $R$. (By Step 1 any scc would have to separate one of the $b\left(s_{i}\right)$ from the others. Thus any component of $R$ hitting $b\left(s_{i}\right)$ cuts a disk off $b S$ contrary to Step 2 while $b\left(s_{i}\right)$ missing $F \cup G$ implies the lemma as we have seen.)

Step 4. If $w$ is an arc in $\partial F$ with both endpoints in the same component of $\partial b S$ and (int $w) \cap b S=\varnothing$, then $b^{-1}(w)$ misses int $P$ where $P$ is the annulus 
in $\partial K$ bounded by $s_{2}$ and $s_{3}$. (Otherwise $b^{-1}(w)$ lies in $P$ and cuts a disk off $P-s_{1}$; that is, $w$ along with an arc $v$ in $\partial b S$ bounds a disk in $\partial K$. Use this disk to pull $v$ to $w$ and then to the other side of $w$. This either reduces the number of components of $R$, a contradiction, or it converts one arc of $R$ into a scc. The scc can be removed as before to also reduce the number of components of $R$, another contradiction.)

Step 5. Let $Q$ denote the annulus in $\partial K$ bounded by $f_{1}$ and $g_{1}$ and let $v$ be an arc in $\partial b S$ with endpoints in $b S \cap F$ and whose interior misses $F \cup G$. Then $v$ misses int $Q$ and both endpoints are in the same component of $\partial F$. (The first part is similar to Step 4 and the second follows from the first and the fact that $g_{1}$ separates $\partial F$ in $\partial K$.)

Step 6. $f_{i}$ hits each component of $\partial b S$ the same number of times and if $f_{i}$ does hit $\partial b S$, then there is an embedding $w$ of the closed interval $[0,5]$ into $f_{i}$ so that $w(0), w(5)$ are in $b\left(s_{2}\right) ; w(1), w(4)$ are in $b\left(s_{1}\right) ; w(2), w(3)$ are in $b\left(s_{3}\right)$; and $w(x)$ is not in $\partial b S$ for any other $x$. (Consider $b^{-1}\left(f_{i}\right)$ and follow it using Step 4.)

Definition. Components of $R$ are said to be separating or nonseparating depending upon whether or not they separate the annulus $F$ or $G$ containing them.

Step 7. There do not exist disks $D$ and $E$ in $b S$ with the following properties: (a) $D \cap E=\partial D \cap \partial E$ is a nonseparating arc in $R$, (b) both $\partial D$ and $\partial E$ are unions of four arcs, two of which are nonseparating arcs of $R$ and the other two are arcs in $\partial b S$, and (c) $F \cup G$ misses the interiors of $D$ and $E$. (See Figure 4.)

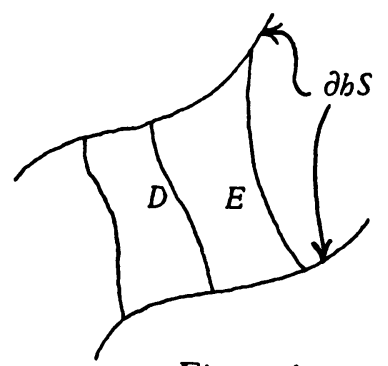

Figure 4

Proof of Step 7. Suppose not and consider the following cases.

Case 1. The nonseparating arcs of $R$ in question all lie in $F$ (similarly if all lie in $G$ ). Then one of the endpoints of $D \cap E$ is in $f_{1}$. Consider the arcs in $\partial b S \cap D$ and $\partial b S \cap E$ with this endpoint and obtain a contradiction to general positioning using Step 5 .

Case 2. $D \cap E$ lies in $G$ and the other two lie in $F$. Let $v_{1}$ be the arc in $\partial D \cap \partial b S$ with one endpoint in $f_{2}$. Since int $v_{1}$ misses $F \cup G$ and the other 
endpoint is in $\partial G$, the endpoint must be in $g_{1}$. If $v_{2}$ is the arc of $\partial E \cap \partial b S$ with this endpoint, then the other endpoint is in $f_{1}$ by Step 5 and general positioning. Let $v_{3}$ be the arc in $\partial D \cap \partial b S$ with one endpoint in $f_{1}$. Since one endpoint of $D \cap E$ is in $g_{1}$, the other is in $g_{2}$; that is, the other endpoint of $v_{3}$ is in $g_{2}$. Now if $v_{4}$ is the other component of $\partial E \cap \partial b S$ (that is, the one with an endpoint in $g_{2}$ ) we have that $v_{3} \cup v_{4}$ is an arc with one endpoint in $f_{1}$ and whose interior misses $\partial F$ but hits $g_{2}$. Thus the other endpoint is also in $f_{1}$. This is impossible since now the arc $E \cap F$ in $R$ has both endpoints in $f_{1}$, contrary to our hypothesis.

Case 3. The two arcs of $\partial D$ in $R$ lie in $F$ and the other arc of $\partial E$ in $R$ lies in $G$. (Follow the arcs starting in $G$ as in Case 2. This contradiction is easier to obtain.)

Step 8. There is no component of $R$ with both endpoints in $b\left(s_{i}\right)$ for $i=1$, 2, 3. (Suppose not and let $u$ be such a component. Because of Step 2, $u$ separates the $b\left(s_{j}\right), j \neq i$. That is, $u$ separates $b S$ into two annuli. Thus any component of $R$ with one endpoint in $b\left(s_{j}\right), j \neq i$, has the other in $b\left(s_{i}\right)$ by Step 2. Hence there are more points in $b\left(s_{i}\right) \cap(\partial F \cap \partial G)$ than in $b\left(s_{j}\right) \cap(\partial F \cap \partial G)$; note $u$ has both endpoints in $b\left(s_{i}\right)$. But Step 6 implies that they are the same size, a contradiction.)

Step 9. No component of $R$ is separating. (If not then consider one $u$ which cuts a disk $E$ off say $F$ such that $E \cap b S=u$. By Step 8, $w=\operatorname{cl}(\partial E-u)$ has endpoints in different components of $\partial h S$. 'Thus $w^{\prime}=b^{-1}(w)$ has endpoints in different $s_{i}$ and since $s_{1}$ separates $s_{2}$ and $s_{3}$ in $\partial K$ one endpoint is in $s_{1}$ and by symmetry we suppose the other is in $s_{2}$. An isotopy on $w^{\prime}$ allows it to be assumed that $w^{\prime}$ hits $f_{1}$ exactly once. Thus $b^{-1}(\partial E) \subseteq S \cup b^{-1}(w)$ and $b^{-1}(\partial E)$ is a scc having linking number \pm 1 with $f_{2}$ in $S^{3}$. But $b^{-1}(\partial E)$ is the boundary of a disk $b^{-1}(E)$ in $K \subseteq S^{3}$ which misses $f_{2}$. This implies the linking number is zero and we have a contradiction.)

Step 10. If $b\left(s_{i}\right)$ hits either $f_{1}$ or $g_{1}$, then it hits the other (Step 5 and general positioning).

Step 11. A contradiction is now obtained. Steps $6,9,10$ require at least twelve components in $R$, and this along with Step 8 leads to a contradiction to Step 7. The proof is complete.

For the two annuli $F$ and $G$, we will define homeomorphisms $f$ and $g$ respectively as follows: cut $K$ at $F$ and twist one component of $F$ in the cut manifold $360^{\circ}$ and glue the manifold back together to form $K$ again. This induces $C$-homeomorphisms at $f_{1}$ and $f_{2}$ in $\partial K$ [2a]. Similarly $g$ is defined for $G$. Let $r$ be the involution which rotates $K$ about the line $l$ in Figure 1. This interchanges $F$ and $G$. $f$ and $g$ could have been so chosen that $r f=g r$. Note that $K$ has an orientation-reversing homeomorphism which consists of reflection through a 
surface perpendicular to each of $F, G$, and $S$. We will therefore consider only those homeomorphisms which are orientation-preserving.

Proof of the theorem. To check that there are no additional relators one needs only to use the given relators to put any relator in the form $r^{k} g^{p} f^{q}$ for integers $k, p, q$ where $k=0,1$ and then observe what happens on $H_{1}(\partial K)$.

Let $b$ be any orientation-preserving homeomorphism such that $b\left(s_{1}\right)=s_{1}$ (Lemma 3). Using regular neighborhood theory and $r$ if necessary we have $b^{\prime} P$ $=P$ and $b^{\prime}\left(s_{i}\right)=s_{i}$ for $i=1,2,3$ where $P$ is the annulus in $\partial K$ bounded by $s_{2}$ and $s_{3}$. Here $b^{\prime}$ is either $b$ or $r b$ composed with an isotopy depending upon whether or not $b$ interchanges the sides of $s_{1}$ in $\partial K$.

Step 1. Since $b^{\prime}$ is orientation-preserving so is $b^{\prime} \mid \partial K$ and since $b^{\prime}$ preserves the sides of $s_{1}$ in $\partial K$ we have that $b^{\prime} \mid s_{1}$ is orientation-preserving and thus we may assume that $b^{\prime} \mid P=1$, the identity. All future isotopies are assumed to be the identity on $P$ unless otherwise specified.

Step 2. An isotopy allows us to assume that $b^{\prime}\left(f_{2}\right)=f_{2}$ and $b^{\prime}\left(g_{2}\right)=g_{2}$. Consider $b^{\prime}\left(f_{2}\right)$. Our construction will not alter $g_{2}$ and consequently we can repeat it for $b^{\prime}\left(g_{2}\right)$ by symmetry without altering $f_{2}=b^{\prime}\left(f_{2}\right)$. By an isotopy which is the identity on $\partial K$ we can put $b^{\prime} F$ and $F$ in general position so that $\left(b^{\prime} F\right) \cap$ $F$ has the minimum number of components possible. scc's in $F \cap b^{\prime} F$ do not bound disks in either $b^{\prime} F$ or $F$ as before. If $b^{\prime}\left(f_{2}\right)$ hits $f_{2}$ there is a disk $D$ in $b^{\prime} F$ cut off by a component $u$ of $F \cap b^{\prime} F$ so that $D \cap F=u$ (note that no component of $F \cap b^{\prime} F$ is an arc with an endpoint in $f_{1}$ since $f_{1} \subseteq P$ ). Then $u$ cuts a disk $E$ off $F$ at $f_{2}$ and thus $E \cup D$ is a properly embedded disk in $K$ missing $P$ and which cuts a 3-cell off $K$. Use this 3-cell to construct an isotopy which is the identity on $P \cup G$ taking $D$ onto $E$ and then slightly to the other side. Since this reduces the number of components of $F \cap b^{\prime} F$ we have that $b^{\prime}\left(f_{2}\right)$ misses $f_{2}$. Cut $\partial K$ at $f_{2}$ and $s_{3}$. Then $b^{\prime}\left(f_{2}\right)$ lies in a disk with two holes and thus is parallel to one of the boundary components. It cannot be the one for $s_{3}$ since $s_{3}$ separates $\partial K$. Thus $b^{\prime}\left(f_{2}\right)$ is parallel to $f_{2}$ in $\partial K$. Also, $b^{\prime}\left(f_{2}\right)$ lies in the complementary domain of $b^{\prime}\left(s_{3}\right)$ in $\partial K$ not containing $b^{\prime} P$ and since $b^{\prime} \mid P$ $=1$ we have that $f_{2}$ and $b^{\prime}\left(f_{2}\right)$ lie in the same complementary domain of $P$ and hence we can construct an isotopy which is the identity on $P \cup G$ and takes $b^{\prime}\left(f_{2}\right)$ onto $f_{2}$.

Step 3. An isotopy allows us to assume that $b^{\prime} F=F$ and $b^{\prime} G=G$. Put $b^{\prime} F$ and $F \cup G$ in general position via an isotopy which is the identity on $\partial K$. Now $b^{\prime} F \cap(F \cup G)$ consists of $\partial F=b^{\prime}(\partial F)$ and scc's in the interior of the sets. Those scc's bounding disks in one of the sets can be eliminated in the usual manner. We eliminate interior pairs in $b^{\prime} F \cap(F \cup G)$ by Lemma 2 and once $b^{\prime} F$ is off the interior of $F \cup G$ we use Lemma 2 to take it onto $F$. Repeat the above process for $G$ requiring the isotopies to be the identity on $F=b^{\prime} F$ as well as on $\partial K$. 
Step 4. An isotopy now allows us to assume $b^{\prime} S=S$ also. An isotopy which is the identity on $\partial K \cup F \cup G$ allows us to push $b^{\prime} S$ into a regular neighborhood of $S$ in $K$. Using general positioning of $b^{\prime} S$ and $S$ and $[5$, p. 65] we can push the components of $\left(b^{\prime} S\right)-S$ to the other side of $S$ one at a time to get $S \cap b^{\prime} S=$ $\partial S$. Again apply $\left[5\right.$, p. 65] to get $b^{\prime} S$ parallel to $S$.

Step 5. We now alter $b^{\prime}$. Note that $b^{\prime} \mid S$ is a homeomorphism of $S$ onto itself which is the identity on $\partial S$. Now any isotopy which is the identity on $\partial S$ can be extended to $K$ by first extending to $\partial K \cup F \cup G$ by the identity and then considering $K$ to be cut at $F \cup G \cup S$. Extend to the rest of $K$ by [1, p. 151]. Using Lemma 3 of $[2 a$, p. 537$]$ we may assume that $b^{\prime} \mid S$ is a sequence of $C$-homeomorphisms and isotopies fixed on $\partial S$. An isotopy on $S$ allows us to assume that $b^{\prime} \mid S$ consists of $C$-homeomorphisms about three disjoint scc's $u_{i}$ in $S$, one parallel to each of the $s_{i}$ via an annulus $A_{i}$. The component of $\partial A_{i}$ in $\partial K$ can be rotated in the appropriate direction so that $b^{\prime} \mid A_{i}=1$. This rotation extends to $K$ so that we may assume $b^{\prime} \mid S=1$. However, this alters $b^{\prime} \mid P$ by $C$-homeomorphisms. The homeomorphisms $f$ and $g$ take care of these. That is, there exist integers $p$ and $q$ so that $k=f^{p} g^{q} b^{\prime}$ has the properties that $k \mid(S \cup P)=1, k F=F$, and $k G=G$.

By symmetry the proof will be complete if we can show that $K \mid L$ is isotopic to the identity via an isotopy which is the identity on $S$. $L$ is the closed complementary domain of $S$ in $K$ containing $G$.

Step 6. It may be assumed that $k \mid g_{2}=1$. This follows since $k G=G$ and $k \mid g_{1}=1$ implies that $k \mid g_{2}$ is also orientation-preserving. The isotopy which allows us to assume $\left.k\right|_{g_{2}}=1$ can be extended to $L$ to be the identity on $S \cup P$.

Step 7. Let $T$ be the closed complementary domain of $s_{2}$ in $\partial L$ containing $g_{2}$. Since $k \mid \partial G=1$ and $k G=G$ we may assume as in Step 5 that $k \mid G$ is a $C$ homeomorphism about a scc in the interior of $G$ which is parallel in $G$ to $g_{2}$ (only one scc is needed here). This can be pushed into $T$ as in Step 5 so that we may assume $k \mid G=1$. Cut $T$ at $g_{2}$ and consider $k$ defined on this disk with two holes. As in Step 5, we may assume $k \mid T$ is a product of three $C$-homeomorphisms, one about a $\operatorname{scc} u$ parallel to $s_{2}$ and the other two, $w$ and $v$, parallel to $g_{2}$ but on different sides of $g_{2}$.

Step 8. Consider the disks $D_{1}$ and $D_{2}$ of Figure 5. $k\left(\partial D_{1}\right)$ is a scc like $\partial D_{1}$ except that $C$-homeomorphisms are formed at $u$ and $v$ with say $m$ and $n$ twists respectively. Thus in $\pi_{1}(L)$ we have that $k_{*}\left(\partial D_{1}\right)=e^{m} b^{n}$. Abelianizing $\pi_{1}(L)$ to $H_{1}(L)$ we have that $e$ maps to zero and $b$ to a generator of $H_{1}(L)$. But $k_{*}\left(\partial D_{1}\right)=1$ in $\pi_{1}(L)$ since $\partial D_{1}$ bounds a disk. Thus $n=0$. Therefore $e^{m}=1$ in $\pi_{1}(K)$. This implies that $a^{m}=1$ which implies that $m=0$ by the Freiheitsatz [3, p. 252]. Considering $D_{2}$ in a similar fashion we have that the number of twists at $w$ is also zero. Thus $k \mid T=1$ and consequently $k \mid \partial L=1$.

Step 9. Now $L$ is a three-cell with two handles and $k \mid \partial L=1$. It is well 


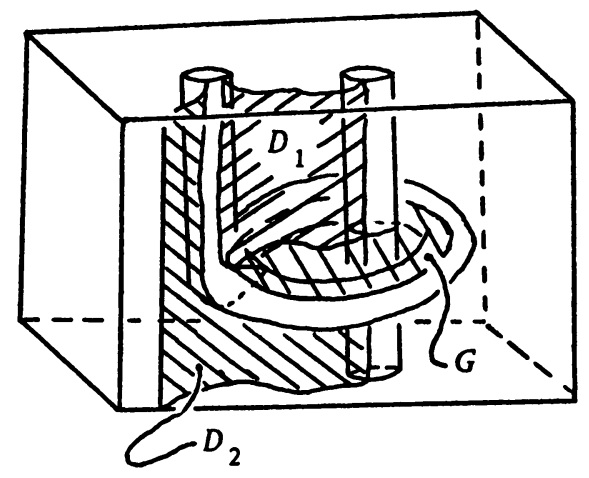

Figure 5

known that such a homeomorphism is isotopic to the identity via an isotopy which is the identity on the boundary. This completes the proof.

3. Other examples. Let $M$ be the manifold which is the closure of the complement in $S^{3}$ of the 1-complex of Figure 6 .

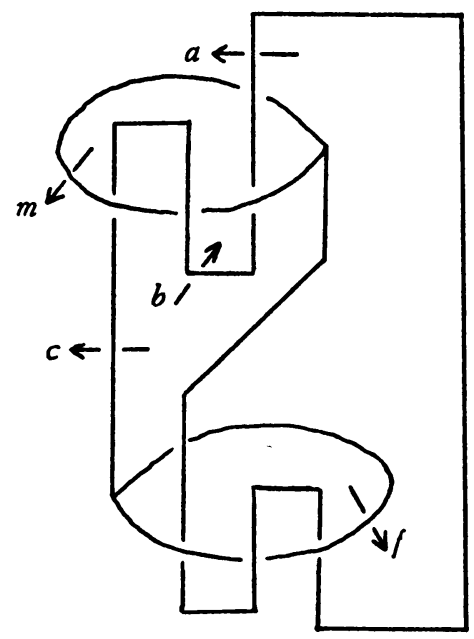

Figure 6

It can be shown that the boundary of any properly embedded annulus in $M$ either bounds an annulus in $\partial M$ or its components bound disjoint disks in $\partial M$. The proof for $M$ uses the same techniques as that for $K$ but the homeomorphisms are a little more difficult to describe. It is easier to describe them algebraically than to do so geometrically although this can also be done. From Figure 6 one can show that $\pi_{1}(M)$ has the presentation: $\left(f, m, b ; m^{-1} b m=f a f a^{-1} f^{-1} a^{-1} f^{-1} a\right)$ where $a=b^{-1} \mathrm{mbm}^{-1} b$. To establish the homeomorphisms on $M$ we define the 
isomorphisms $R$ and $S$ of $\pi_{1}(M)$ onto itself by $R(f)=a^{-1} f a^{3}, R(m)=m c^{-2}, R(b)$ $=b^{-1}$, and $S(f)=b m^{-1} b, S(m)=f a^{-1} f^{-1} a^{-1} f^{-1}, S(b)=f a^{-1} f^{-1}$. Using Corollary 6.5 of $[5$, p. 80] we obtain homeomorphisms of $M$ onto itself. The one for $R$ is analogous to $r$ of the theorem in $\$ 2$ and the one for $S$ is analogous to the orientation-reversing homeomorphism on $K$ mentioned prior to the proof of the theorem. See [3] for the necessary combinatorial group theory.

Let $M^{\prime}$ be the manifold which is the closure of the complement of a regular neighborhood in $S^{3}$ of the 1-complex of Figure 7.

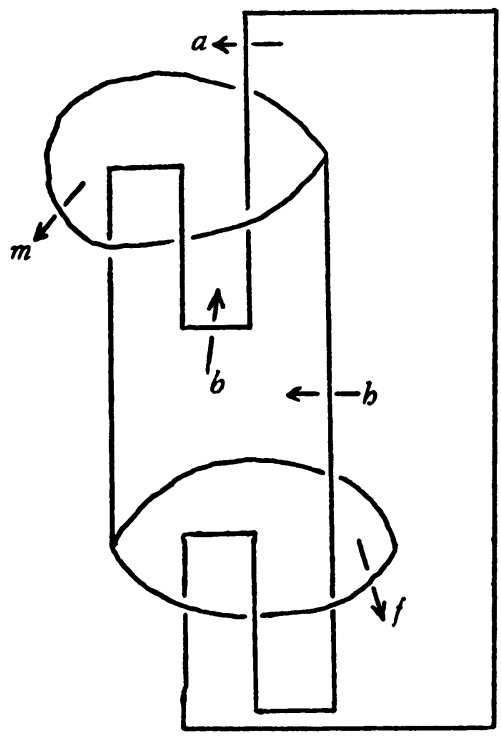

Figure 7

Let $S$ be a disk with two holes in $M$ analogous to the disk with two holes $S$ in $K$. Let $G$ and $F$ be disks with two holes in $M$ analogous to the annuli $G$ and $F$ respectively in the manifold $K . M^{\prime}$ can be thought of as replacing the complementary domain of $S$ in $M$ containing $G$ by a copy of the one containing $F$ so that the symmetry that gave rise to $r$ in the theorem of $\$ 2$ is present in $M^{\prime}$. We define an isomorphism $T: \pi_{1}(M) \rightarrow \pi_{1}\left(M^{\prime}\right)$ by $T(m)=m, T(b)=b, T(f)=f^{-1} b^{-1} f b^{-1} f$ using the notation of Figures 6 and 7. This will induce a homeomorphism of $M$ onto $M^{\prime}[5, \mathrm{p} .80]$. This orientation-preserving homeomorphism does not extend to $S^{3}$.

As a final example we include the manifold $N$, the closure of the complement of a regular neighborhood of the 1-complex of Figure 8.

It can be shown that every homeomorphism of $N$ onto itself is isotopic to the identity; in particular there are no orientation-reversing homeomorphisms of $N$ onto itself. If the corresponding sets $F, G$, and $S$ are defined in $N$ the proof brings 


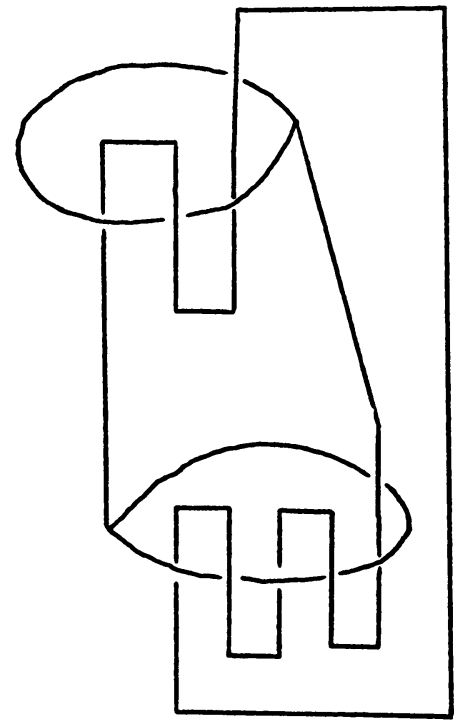

Figure 8

each of their homeomorphic images back onto themselves. The three holes of $G$ will force $b \mid \partial G$ to be orientation-preserving.

\section{BIBLIOGRAPHY}

1. J. F. P. Hudson, Piecewise linear topology, University of Chicago Lecture Notes prepared with the assistance of J. L. Shaneson and J. Lees, Benjamin, New York, 1969. MR 40 \#2094.

2. a) W. B. R. Lickorish, A representation of orientable combinatorial 3-manifolds, Ann. of Math. (2) 76 (1962), 531-540. MR $27 \# 1929$.

b) - Homeomorphisms of non-orientable two-manifolds, Proc. Cambridge Philos. Soc. 59 (1963), 307-317. MR $26 \# 3029$.

c) - A finite set of generators for the homeotopy group of a 2 manifold, Proc. Cambridge Philos. Soc. 60 (1964), 769-778. MR $30 \# 1500$.

3. Wilhelm Magnus, Abraham Karrass and Donald Solitar, Combinatorial group theory: Presentations of groups in terms of generators and relations, Pure and Appl. Math., vol. 13, Interscience, New York, 1966. MR 34 \#7617.

4. Donald Myers, Homeomorphisms on cubes with holes, Ph.D. Thesis, University of Iowa, Iowa City, Ia., August, 1971.

5. Friedhelm Waldhausen, On irreducible 3 -manifolds which are sufficiently large, Ann. of Math. (2) 87 (1968), 56-88. MR 36 \#7146. 53201

DEPARTMENT OF MATHEMATICS, UNIVERSITY OF VISCONSIN, MILTAUKEE, WISCONSIN

Current address: 11837 Diane Drive, Wauwatosa, Wisconsin 53226 